\title{
Alocação de Espectro com Redução de Interferências entre Circuitos em Redes Ópticas Elásticas
}

\author{
Alexandre Fontinele ${ }^{1}$, Iallen Santos ${ }^{1,3}$, Jurandir Lacerda Jr. ${ }^{4}$, \\ André Soares $^{2}$, José Suruagy Monteiro ${ }^{1}$ e Divanilson R. Campelo ${ }^{1}$ \\ ${ }^{1}$ Centro de Informática (CIn) - Universidade Federal de Pernambuco (UFPE) \\ Recife - PE - Brasil \\ ${ }^{2}$ Departamento de Computação - Universidade Federal do Piauí (UFPI) \\ Teresina - PI - Brasil \\ ${ }^{3}$ Instituto Federal de Educação, Ciência e Tecnologia do Piauí (IFPI) \\ São Raimundo Nonato - PI - Brasil \\ ${ }^{4}$ Instituto Federal de Educação, Ciência e Tecnologia do Piauí (IFPI) \\ Corrente - PI - Brasil \\ acf3ecin.ufpe.br
}

\begin{abstract}
This paper proposes a new spectrum assignment algorithm in elastic optical networks. The goal of the proposed algorithm is to allocate spectrum bands that cause less interference to the quality of transmission of the other circuits. A performance evaluation study was carried out comparing the proposed algorithm with eight other spectrum assignment algorithms presented in the literature. Results of the performance evaluation study show that the proposed algorithm presented a superior performance to the other algorithms in terms of circuit blocking probability and bandwidth blocking probability for the scenarios evaluated. In general, the proposed algorithm presented a minimum gain of $28.92 \%$ in terms of circuit blocking probability and a minimum gain of $3.63 \%$ in terms of bandwidth blocking probability.
\end{abstract}

Resumo. Este artigo propõe um novo algoritmo de alocação de espectro em redes ópticas elásticas. O objetivo do algoritmo proposto é alocar faixas de espectro que causem menos interferências na qualidade de transmissão dos outros circuitos. Foi realizado um estudo de avaliação de desempenho comparando o algoritmo proposto com outros oito algoritmos de alocação de espectro apresentados na literatura. Resultados do estudo de avaliação de desempenho mostram que o algoritmo proposto apresentou um desempenho superior aos outros algoritmos em termos de probabilidade de bloqueio de circuito e probabilidade de bloqueio de banda para os cenários avaliados. Em geral, o algoritmo proposto apresentou um ganho mínimo de $28,92 \%$ em termos de probabilidade de bloqueio de circuito e um ganho mínimo de 3,63\% em termos de probabilidade de bloqueio de banda.

\section{Introdução}

Os serviços emergentes (tais como realidade virtual/realidade aumentada, rede móvel 5G, serviços de vídeo de alta definição, serviços de computação em nuvem, etc.) têm 
estimulado o desenvolvimento e transformação das tecnologias de comunicação óptica [Wu et al. 2017, Zhu et al. 2017]. As redes ópticas elásticas, com base na tecnologia de multiplexação por divisão de frequência ortogonal (Orthogonal Frequency Division Multiplexing - OFDM), têm atraído o interesse de pesquisas nos últimos anos. Pois podem melhorar significativamente a eficiência espectral da camada óptica com alocação flexível de largura de banda [Chatterjee et al. 2015]. Essa arquitetura de rede óptica permite a divisão do espectro óptico em pequenos intervalos de frequência chamados de slots. Assim, cada circuito pode alocar uma quantidade diferente de slots dependendo da sua demanda de largura de banda.

Para o estabelecimento de um circuito óptico em uma rede óptica elástica é necessário resolver o problema de roteamento, seleção de formato de modulação e alocação de espectro (Routing, Modulation Level, and Spectrum Assignment - RMLSA) [Christodoulopoulos et al. 2011]. O problema RMLSA consiste em i) definir uma rota para um par de nós origem e destino; ii) selecionar um formato de modulação adequado para essa rota e iii) escolher uma faixa de espectro livre (conjunto de slots contíguos e contínuos) na rota definida.

Nas redes ópticas elásticas transparentes, objeto de investigação deste trabalho, os dados são transmitidos da fonte para o destino como um sinal óptico. Toda a operação de roteamento é realizada no domínio óptico sem qualquer conversão para o domínio eletrônico. O sinal óptico sofre degradação à medida que se propaga da origem para o destino devido às imperfeições da camada física. $\mathrm{O}$ acúmulo das degradações no sinal óptico ao longo de uma rota pode tornar a taxa de erro de bit (Bit Error Rate - BER) intolerável no receptor, inviabilizando a comunicação [Beyranvand and Salehi 2013]. Nesse contexto, algoritmos que levam em consideração as imperfeições de camada física tornam-se mais apropriados para encontrar uma solução para o problema RMLSA.

Este artigo apresenta um novo algoritmo de alocação de espectro ciente de imperfeições da camada física em redes ópticas elásticas, chamado de Spectrum Assignment with Interference Reduction (SAIR). O algoritmo proposto busca selecionar faixas de espectro livre reduzindo a interferência na qualidade de transmissão (Quality of Transmission - QoT) dos outros circuitos já ativos na rede. É também apresentado um estudo de avaliação de desempenho comparando o algoritmo proposto com o estado da arte. Os resultados mostram que o algoritmo proposto obteve um ganho mínimo de $28,92 \%$ em termos de probabilidade de bloqueio de circuito e um ganho mínimo de 3,63\% em termos de probabilidade de bloqueio de banda.

As principais contribuições deste trabalho são: avaliar os algoritmos de alocação de espectro comumente utilizados pela literatura e do estado da arte em um cenário mais realista em comparação aos cenários em que eles foram avaliados até o presente momento; E propor um novo algoritmo de alocação de espectro ciente dos efeitos de camada física em redes ópticas elásticas.

As demais seções deste artigo estão organizadas da seguinte forma. A Seção 2 apresenta o problema de alocação de espectro. A Seção 3 discute os trabalhos relacionados. Considerações sobre os efeitos de camada física são discutidas na Seção 4. O algoritmo proposto é apresentado na Seção 5. A Seção 6 apresenta um estudo de avaliação de desempenho. Por fim, as conclusões do trabalho são destacadas na Seção 7. 


\section{Problema de Alocação de Espectro}

O problema RMLSA é um problema NP-completo [Christodoulopoulos et al. 2011]. Os algoritmos propostos para resolver o problema RMLSA podem ser divididos em duas categorias: integrados e sequenciais [Chatterjee et al. 2015]. Os algoritmos sequenciais subdividem o problema RMLSA em subproblemas e resolvem cada subproblema em etapas distintas, uma após a outra. Os algoritmos integrados buscam resolver o problema RMLSA em uma única etapa, realizando o roteamento, seleção de formato de modulação e a alocação de espectro simultaneamente. Resolver o problema RMLSA de forma sequencial é computacionalmente mais simples do que resolver de forma integrada.

O algoritmo de alocação de espectro é o responsável por escolher a faixa de slots adequada para o estabelecimento do circuito óptico requisitado. Estes slots devem ser alocados adjacentes uns aos outros para atender a restrição de contiguidade de espectro. Além disso, a continuidade desses slots em cada enlace da rota deve ser garantida. Se a requisição solicita $t$ unidades de espectro, então $t$ slots contíguos devem ser alocados para a requisição de circuito (devido à restrição de contiguidade de espectro). Os mesmos $t$ slots contíguos devem ser alocados em cada enlace ao longo da rota (devido a restrição de continuidade do espectro) [Chatterjee et al. 2015].

A alocação de slots para as requisições de circuitos e sua liberação após o término das transmissões pode provocar a fragmentação do espectro. A fragmentação é um conjunto de slots livres não contíguos entre si. O problema da fragmentação do espectro influencia diretamente na eficiência das redes ópticas elásticas [Chatterjee et al. 2015]. Na Figura 1 é apresentado um exemplo de fragmentação do espectro.

a)

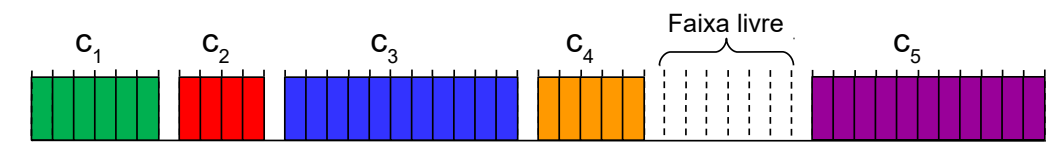

b)

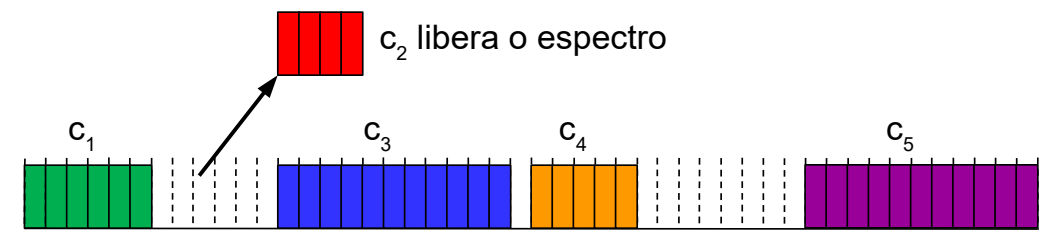

c)

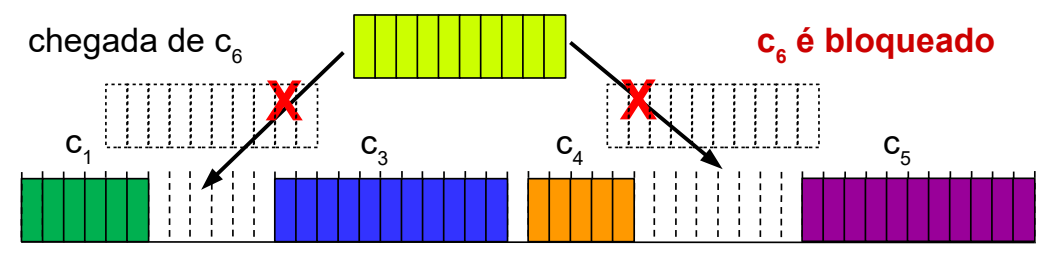

Figura 1. Cenário com fragmentação do espectro.

Na Figura 1(a) é apresentado um cenário com cinco circuitos já estabelecidos (slots coloridos) e uma faixa de slots livres. O slot livre entre os circuitos é chamado de banda de guarda [Chatterjee et al. 2015]. Em um dado momento o circuito $c_{2}$ libera os slots que estava utilizando, ficando agora duas faixas de slots livres (Figura 1(b)). A Figura 1(c) apresenta o momento da chegada da requisição do circuito $c_{6}$. O circuito $c_{6}$ precisa de dez slots para ser estabelecido, mas acaba sendo bloqueado porque as faixas de 
slots livres não possuem slots contíguos suficientes para o estabelecimento de $c_{6}$.

\section{Trabalhos Relacionados}

O problema RMLSA é tratado de forma sequencial neste trabalho, com foco na resolução do subproblema de alocação de espectro. Para o subproblema de alocação de espectro existem várias heurísticas apresentadas pela literatura e cada uma delas pode ser utilizada em conjunto com qualquer estratégia de roteamento e seleção de formato de modulação [Chatterjee et al. 2015]. Nesta seção são apresentadas as principais heurísticas de alocação de espectro relacionadas com a proposta deste trabalho.

Dentre os métodos de alocação de espectro de fácil implementação e com baixa complexidade computacional (ver na Seção 5) temos o FirstFit, RandomFit, LastFit, BestFit e ExactFit [Rosa et al. 2012, Leiva et al. 2014, Chatterjee et al. 2015]. O método FirstFit é o mais utilizado na literatura. Nele, os slots são indexados e mantidos em uma lista com os slots disponíveis. No momento do estabelecimento de um circuito é escolhida uma faixa de slots disponíveis com os menores índices presentes na lista. Assim como no FirstFit, o método RandomFit, também mantém uma lista com os slots disponíveis. Porém no momento do estabelecimento de um circuito ele busca escolher aleatoriamente uma faixa de slots disponíveis.

O método LastFit escolhe uma faixa de slots disponíveis com os maiores índices presentes na lista de slots disponíveis. O método BestFit escolhe a faixa de slots disponíveis cuja a quantidade de slots é a mais próxima da quantidade de slots requisitadas. $\mathrm{O}$ método ExactFit busca alocar uma faixa de slots livres com a quantidade de slots igual à solicitada para o estabelecimento do novo circuito. Caso contrário, busca alocar a faixa com a maior diferença entre slots livres e solicitados.

A Figura 2 apresentada um cenário para a demonstração da aplicação dos métodos de alocação de espectro. Considera-se a chegada de uma requisição de circuito com largura de banda de $40 \mathrm{Gbps}$, rota com $3000 \mathrm{~km}$ de distância e que requer três slots. Na Figura 2, as faixas de slots coloridos representam slots já ocupados por circuitos $\left(c_{i}\right)$. As faixas de slots livres (contíguos e contínuos) que são suficientes para acomodar o estabelecimento do novo circuito estão representadas por números.

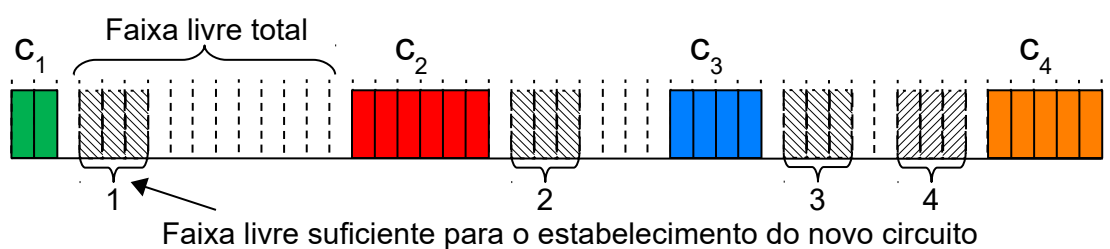

Figura 2. Cenário para a demonstração da aplicação dos métodos de alocação de espectro.

Aplicando os métodos de alocação de espectro já discutidos no cenário apresentado pela Figura 2 tem-se as seguintes escolhas. O método FirstFit seleciona a faixa de slots livres representada pelo número 1. O método RandomFit, para o exemplo, aloca aleatoriamente a faixa de slots livres representada pelo número 3. E o método LastFit escolhe a faixa de slots livres representada pelo número 4. Enquanto o método BestFit seleciona a faixa de slots livres representada pelo número 2. Já o método ExactFit não 
encontra uma faixa de slots livres total com o mesmo número de slots solicitados. Então a faixa de slots livres selecionada pelo ExactFit é a representada pelo número 1.

Alguns trabalhos propõe a combinação de FirstFit e LastFit (FirstLastFit) para evitar a fragmentação do espectro [Talebi et al. 2014, Chatterjee et al. 2015, Liu et al. 2017]. Nesses trabalhos os slots nos enlaces são divididos em partições. O método FirstFit sempre tenta escolher os slots livres com os menores índices na partição de número ímpar. Enquanto o método LastFit escolhe os slots livres com os maiores índices na partição de número par. Quando chega uma requisição de circuito é utilizado algum mecanismo para decidir em qual partição será selecionada a faixa de slots livres para o estabelecimento do circuito. Geralmente o mecanismo de decisão é baseado nas larguras de banda solicitadas pelas requisições. Por exemplo, em um cenário que as granularidades de larguras de banda requisitadas são 10, 40, 60, 80 e 100 Gbps. As requisições de larguras de banda 80 e 100 Gbps seriam alocadas na partição que utiliza o FirstFit. Enquanto as requisições de larguras de banda 10, 40 e 60 Gbps seriam alocadas na partição que utiliza o LastFit. Aplicando o FirstLastFit no cenário apresentado pela Figura 2, a faixa de slots livres selecionada é a representada pelo número 4.

Os autores em [Beyranvand and Salehi 2013] propõem um método de alocação de espectro chamado de Traffic Balancing Spectrum Assignment (TBSA). O método TBSA busca alocar os slots de frequência de forma a distribuir a carga de tráfego sobre o espectro. O TBSA busca pelos intervalos de espectro livres que possuem quantidade de slots maior ou igual à quantidade de slots solicitados para o estabelecimento do circuito. Depois divide os intervalos encontrados em duas partes, chamadas de $W_{1}$ e $W_{2}$. Verifica a quantidade de slots em cada parte e seleciona a parte que possuir mais slots. Repete o processo de divisão, contagem e seleção até que a parte $W_{i}$ que sobrar contenha apenas um intervalo.

Aplicando o TBSA no cenário da Figura 2, ele consideraria as duas primeiras faixas livres totais como sendo a parte $W_{1}$ e terceira faixa livre total como sendo a parte $W_{2}$. O TBSA selecionaria a parte $W_{1}$ para continuar a investigar, já que $W_{1}$ possui mais slots livres que $W_{2}$. Depois a primeira faixa livre total passaria a representar a nova parte $W_{1}$ e segunda faixa livre total seria a nova parte $W_{2}$. A parte $W_{1}$ seria selecionada para dar continuidade no processo de busca por possui mais slots livres que $W_{2}$. Por fim, o método TBSA seleciona a faixa de slots livre representada pelo número 1 para o estabelecimento do novo circuito.

Os autores em [Chatterjee et al. 2016] propõem um novo algoritmo de alocação de espectro baseado na combinação de FirstFit, LastFit e ExactFit (FirstLastExactFit). O objetivo da proposta é aumentar o número de slots disponíveis (contíguos e contínuos) e evitar pequenas quantidades de slots disponíveis contíguos que são gerados quando esses métodos são aplicados separadamente. O FirstLastExactFit utiliza a definição de circuitos disjuntos e não disjuntos para decidir qual método de alocação de espetro vai ser aplicado. Circuitos com rotas disjuntas são alocados usando o método FirstExactFit. Enquanto circuitos com rotas não disjuntas são alocados usando o método LastExactFit. Os autores apresentam uma heurística para a criação do grupo de circuitos disjuntos. No exemplo da Figura 2, se o novo circuito for classificado como não pertecente ao grupo de circuitos disjuntos, a faixa de slots livres selecionada é a representada pelo número 4. 
Em [Chatterjee and Oki 2016], os autores propõem um algoritmo de alocação de espectro ciente dos efeitos de camada física. O método chamado de Dispersion Adaptive First Last Fit (DAFLF) busca alocar espectro reduzindo o efeito de dispersão. O método DAFLF utiliza um parâmetro chamado de $\beta$ para determinar se a alocação de espectro vai utilizar o FirstFit ou o LastFit. Se a distância do circuito requisitado for maior ou igual ao valor de $\beta$ é aplicado o FirstFit. Caso contrário é aplicado o LastFit. Depois de decidir qual método de alocação de espectro vai ser utilizado, o algoritmo busca a faixa de slots livres que acomoda a quantidade de slots requisitados e que possua QoT aceitável. Considerando que o $\beta$ seja igual a $1000 \mathrm{~km}$ para o cenário apresentado pela Figura 2, o DAFLF escolhe a faixa de slots livres representado pelo número 1.

\section{Considerações sobre os Efeitos de Camada Física}

O sinal óptico ao ser transmitido por enlaces e dispositivos ópticos perde a sua qualidade devido aos efeitos de camada física. As degradações causadas pelos efeitos de camada física são geralmente classificadas em duas categorias: efeitos lineares e efeitos não lineares. Na primeira categoria estão os efeitos que são independentes da potência do sinal e afetam cada circuito separadamente. Os efeitos presentes na segunda categoria são fortemente dependentes da potência acumulada e da potência individual dos circuitos ópticos transportados em paralelo na mesma fibra, causando interferências nos circuitos individualmente e entre eles [Saradhi and Subramaniam 2009].

O efeito linear que contribui significativamente para limitar o alcance de transmissão do sinal óptico é a emissão espontânea amplificada (Amplified Spontaneous Emission - ASE) gerada nos amplicadores ópticos. E os principais efeitos não lineares são AutoModulação de Fase (Self-Phase Modulation - SPM), Modulação de Fase Cruzada (CrossPhase Modulation - XPM) e Mistura de Quadro Ondas (Four-Wave Mixing - FWM) [Beyranvand and Salehi 2013].

Os efeitos de camada física podem impactar significativamente na qualidade do sinal óptico tornando-a inaceitável no receptor [Beyranvand and Salehi 2013]. Neste sentido, se a BER chegar a níveis elevados a qualidade de transmissão será impactada. Com isso, poderá gerar um bloqueio por QoT inaceitável. Os receptores ópticos possuem uma curva de desempenho que associa a relação sinal ruído (Signal to Noise Ratio - SNR) diretamente com a BER. Portanto, a SNR pode ser usada como critério de QoT de camada física de um circuito óptico. Detalhamentos sobre o cálculo de SNR adotado neste trabalho podem ser encontrados em [Fontinele et al. 2017].

Caso os níveis de SNR não sejam adequados, a requisição pode ser bloqueada por QoTN (QoT no novo circuito) ou QoTO (QoT nos outros circuitos já estabelecidos) [Fontinele et al. 2017]. O QoTN é o bloqueio sofrido caso a nova requisição não atinja os níveis adequados de QoT. Mesmo que uma nova requisição atinja tal requisito, ela ainda poderá sofrer bloqueio caso o estabelecimento da nova requisição impacte na QoT dos circuitos já estabelecidos, ocasionando assim o QoTO.

\section{Algoritmo Proposto}

O algoritmo de alocação de espectro proposto neste trabalho é chamado de SAIR. O algoritmo SAIR busca alocar uma faixa de slots livres que causa menos interferência na qualidade de transmissão dos outros circuitos já ativos na rede. O algoritmo SAIR utiliza a 
estratégia de particionamento do espectro, como apresentado na Seção 3. E o mecanismo de decisão também é baseado nas larguras de banda solicitadas pelas requisições de circuitos. A Figura 3 apresenta o fluxograma do algoritmo SAIR. O parâmetro larger Band (destacado pelo número 1 na Figura 3) do algoritmo SAIR representa a largura de banda limite para o mecanismo de decisão.

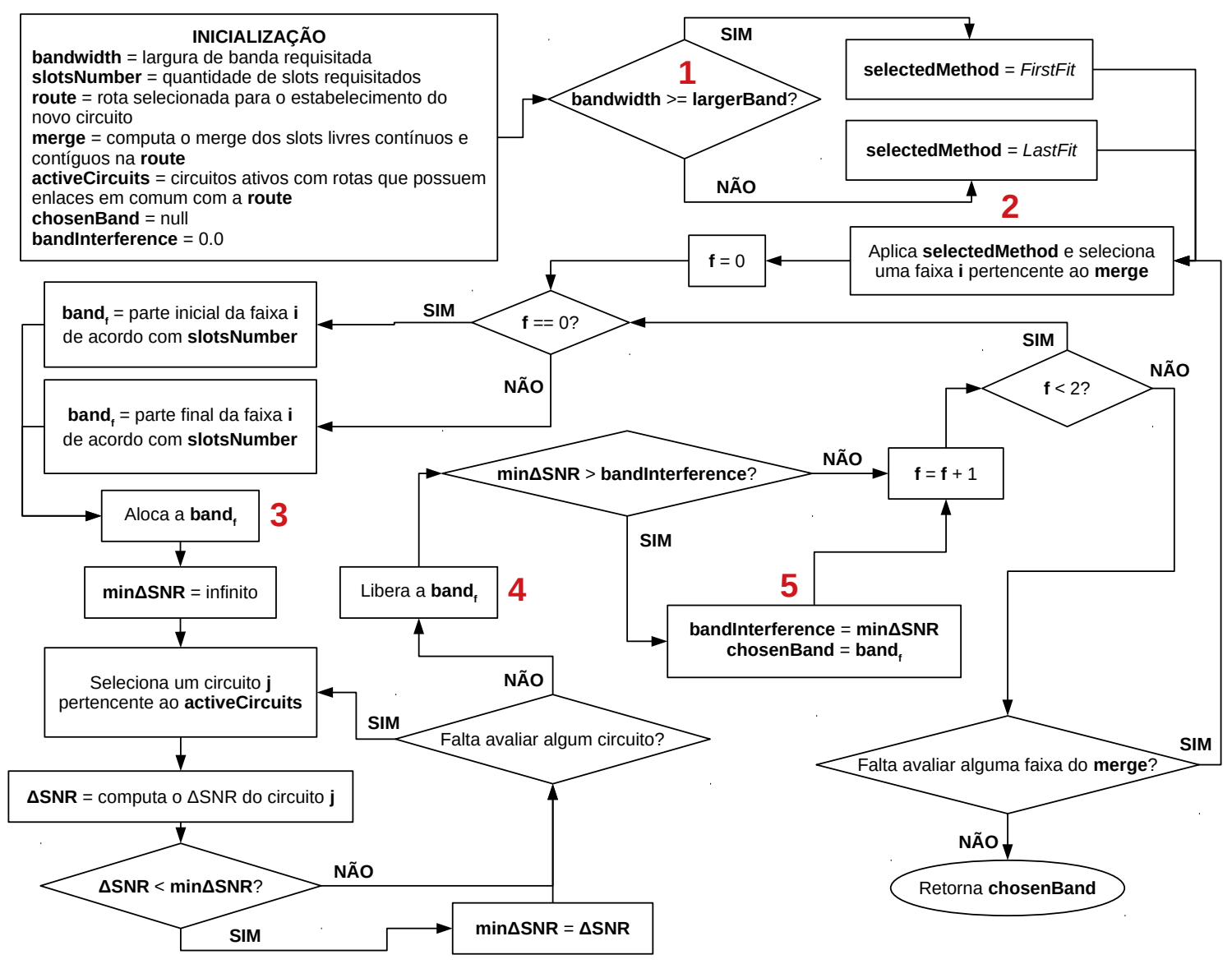

Figura 3. Fluxograma do algoritmo SAIR.

A Figura 4 apresentada um cenário para a demonstração da aplicação do algoritmo SAIR, mesmo cenário apresentado na Seção 3. Considera-se a chegada de uma requisição de circuito $\left(c_{5}\right)$ com largura de banda de 40 Gbps e que precisa de três slots. Na Figura 4, as faixas de slots coloridos representam slots já ocupados por circuitos $\left(c_{i}\right)$. As faixas de slots livres (contíguos e contínuos) que são suficientes para acomodar o estabelecimento do novo circuito estão representadas por números. O valor adotado para largerBand é 80 Gbps (O exemplo apresentado na Figura 6(b) mostra como é selecionado esse valor).

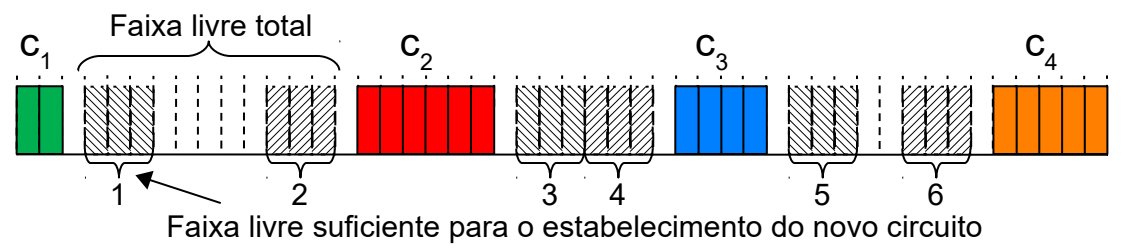

Figura 4. Cenário para a demonstração da aplicação do algoritmo SAIR. 
Aplicando o SAIR no cenário da Figura 4, ele vai procurar a faixa de slots livres que causa menos interferência nos circuitos $c 1, c 2, c 3$ e $c 4$. Se o algoritmo aplicar o método FirstFit as faixas de slots livres serão avaliadas na seguinte ondem: 1, 2, 3, 4, 5 e 6. No caso do algoritmo aplicar o método LastFit as faixas de slots livres serão avaliadas na seguinte ondem: $6,5,4,3,2 \mathrm{e} 1$. Por $c_{5}$ possuir largura de banda inferior a larger Band o método LastFit será selecionado para ser aplicado nas faixas de slots livres (destacado pelo número 2 na Figura 3). Neste passo, o algoritmo apenas seleciona as faixas de slots livres que possuem a quantidade de slots maior ou igual ao número de slots solicitados por $c_{5}$.

No passo destacado pelo número 3 na Figura 3, o novo circuito $c_{5}$ é preestabelecido para verificar a sua interferência nos outros circuitos já ativos. E no passo destacado pelo número 4 na Figura 3, os recursos utilziados por $c_{5}$ são liberados. A interferência é medida através do valor de $\Delta$ SNR dos circuitos ativos que possuem rotas com enlaces em comum com a rota seleciona para o estabelecimento de $c_{5}$. O $\Delta$ SNR representa a diferença entre o SNR do circuito e o limiar de SNR do formato de modulação utilizado pelo circuito. A faixa de slots livres que causar a menor interferência nos outros circuitos é a selecionada (chosenBand) para o estabelecimento do novo circuito (destacado pelo número 5 na Figura 3). Faixas de slots livres que causam interferências significativas nos outros circuitos já ativos (que tornam a QoT inaceitável) não são selecionadas.

A complexidade de tempo dos algoritmos de alocação de espectro FirstFit, LastFit, BestFit, ExactFit, RandomFit, FirstLastFit, TBSA e DAFLF é igual a $O(|E| *|S|)$, em que $E$ é o conjunto de enlaces da rede e $S$ é o conjunto de slots em cada enlace da rede [Chatterjee et al. 2015, Chatterjee and Oki 2016, Liu et al. 2017]. O algoritmo FirstLastExactFit possui duas fases [Chatterjee et al. 2016]. Na primeira fase o algoritmo cria o grupo de circuitos disjuntos. Essa primeira fase pode ser executada apenas uma vez se o conjunto de rotas para todos os pares já forem predeterminadas. Assim, a complexidade de tempo da primeira fase é igual a $O(|P| *|R|)$, em que $P$ é o conjunto de pares de nós origem e destino da rede e $R$ é o conjunto de rotas por par. Na segunda fase é verificado se o circuito em estabelecimento pertence ou não ao grupo de circuitos disjuntos e é aplicado o método apropriado como foi explicado na Seção 3. A complexidade de tempo da segunda fase é igual a $O(|E| *|S|)$ [Chatterjee et al. 2016].

A complexidade de tempo do algoritmo SAIR é igual a $O(|S| *(|E|+|C|))$, em que $C$ é o conjunto de circuitos ativos que possuem rotas com enlaces em comum com a rota selecionada para o estabelecimento de um dado circuito. A estratégia aplicada pelo algoritmo SAIR para a seleção da faixa de slots livres contribui significativamente para o aumento do tempo de execução do algoritmo. Observa-se que a complexidade de tempo do algoritmo SAIR é superior à dos outros algoritmos de alocação de espectro apresentados neste trabalho.

\section{Avaliação de Desempenho}

As métricas consideradas no estudo de avaliação de desempenho dos algoritmos de alocação de espectro foram i) probabilidade de bloqueio de circuito (PBC); ii) probabilidade de bloqueio de banda (PBB) e iii) taxa média de fragmentação externa (TMFE). A taxa média de fragmentação externa mede o nível médio de fragmentação externa de todos os enlaces da rede durante o tempo de simulação. Para medir o nível de fragmentação 
externa foi utilizada a Equação 1 [Rosa et al. 2012].

$$
F_{\text {ext }}=1-\frac{\text { largestFreeBlock }}{\text { totalFree }} \text {. }
$$

Na Equação 1, largestFreeBlock é a quantidade de slots do maior espaço contíguo livre e totalFree é a quantidade total de slots disponíveis. Quando a fragmentação externa está próxima a um (100\%) significa que o espaço disponível está todo dividido em pequenos fragmentos. Em caso do espaço estar todo disponível a fragmentação externa é $0 \%$.

O algoritmo proposto SAIR foi comparado com os algoritmos FirstFit (FF), BestFit (BF), ExactFit (EF), RandomFit (RF), FirstLastFit (FLF), FirstLastExactFit (FLEF), TBSA e DAFLF. O algoritmo LastFit não foi considerado na comparação por apresentar o mesmo desempenho do algoritmo FirstFit. Como foco deste trabalho é a avaliação de desempenho dos algoritmos de alocação de espectro, para o roteamento e seleção de formato de modulação foram adotados algoritmos comumente utilizados na literatura. $\mathrm{O}$ algoritmo de menor caminho de Dijkstra foi utilizado para o roteamento. E para a seleção de formato de modulação foi utilizado o algoritmo de seleção de modulação pela QoT apresentado em [Fontinele et al. 2017]. Assim, os próprios algoritmos de alocação de espectro são os responsáveis pelos seus desempenhos.

As simulações foram realizadas usando o simulador SNetS (SLICE Network Simulator) [Fontinele et al. 2017]. Em cada simulação foram geradas 100000 requisições de circuitos ópticos com sete níveis de granularidade: 10, 40, 80, 100, 160, 200 e 400 Gbps, com as proporções 7:6:5:4:3:2:1, respectivamente. A geração de requisições segue a distribuição de Poisson com a carga de tráfego distribuída uniformemente entre todos os pares de nós origem e destino. Para cada simulação foram realizadas dez replicações com diferentes sementes de geração de variável aleatória. Todos os resultados possuem nível de confiança de 95\%. Foram escolhidas duas topologias reais (NSFNet (National Science Foundation Network) e Pacific Bell) que apresentaram cenários diferentes para o estudo de avaliação de desempenho, Figura 5 [Chaves et al. 2012, Fontinele et al. 2017].
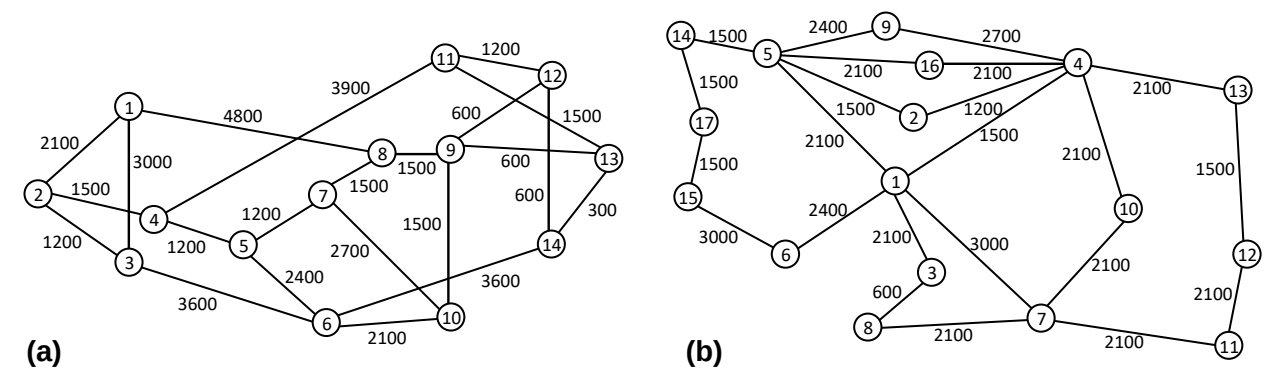

\section{Figura 5. Topologias (a) NSFNet e (b) Pacific Bell. 0 número em cada enlace corresponde a distância em km.}

Os formatos de modulação considerados neste estudo foram BPSK, QPSK, 8QAM, 16QAM, 32QAM e 64QAM. Todos os enlaces da rede são bidirecionais e possuem largura de banda do espectro dividida em 400 slots de frequência. Um slot de frequência possui largura de banda de 12,5 GHz. Os ganhos dos amplificadores são ajustados para compensar as perdas dos dispositivos e da fibra. Outros parâmetros utilizados nas simulações estão listados na Tabela 1 [Fontinele et al. 2017]. 
Tabela 1. Parâmetros de camada física utilizados nas simulações.

\begin{tabular}{c|c}
\hline Descrição & Valor \\
\hline Densidade espectral de potência do sinal & $-17 \mathrm{dBm} / \mathrm{GHz}$ \\
Atenuaçao da fibra $(\alpha)$ & $0,2 \mathrm{~dB} / \mathrm{km}$ \\
Parâmetro de dispersão da fibra $\left(\beta_{2}\right)$ & $16 \mathrm{ps}^{2} / \mathrm{km}$ \\
Coeficiente não linear da fibra $(\gamma)$ & $1,3(\mathrm{Wkm})^{-1}$ \\
Tamanho de um span $\left(L_{s}\right)$ & $100 \mathrm{~km}$ \\
Figura de ruído do amplificador $(N F)$ & $6 \mathrm{~dB}$ \\
\hline
\end{tabular}

Os algoritmos DAFLF e FLF possuem parâmetros internos que influenciam nos seus desempenhos. Por esse motivo foi realizado um estudo para encontrar os melhores valores para os parâmetros internos dos algoritmos DAFLF e FLF. A Figura 6 apresenta os resultados dos estudos realizados com os algoritmos DAFLF e FLF. Na Figura 6(a) são apresentadas as PBC alcançadas com o DAFLF nas topologias NSFNet e Pacific Bell em função do valor utilizado para o $\beta$ (parâmetro interno do DAFLF). Observa-se na Figura 6(a) que o desempenho do DAFLF muda de acordo com o valor utilizado para o $\beta$. Os valores para o $\beta$ que geraram as menores $\mathrm{PBC}$ foram $3500 \mathrm{~km}$ e $5000 \mathrm{~km}$ para as topologias NSFNet e Pacific Bell, respectivamente.

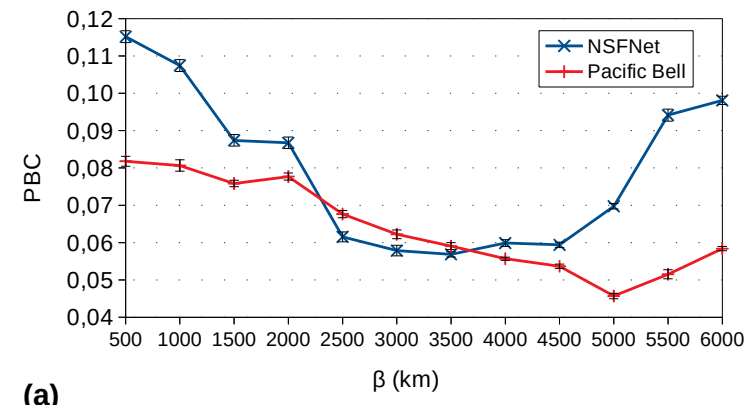

(a)

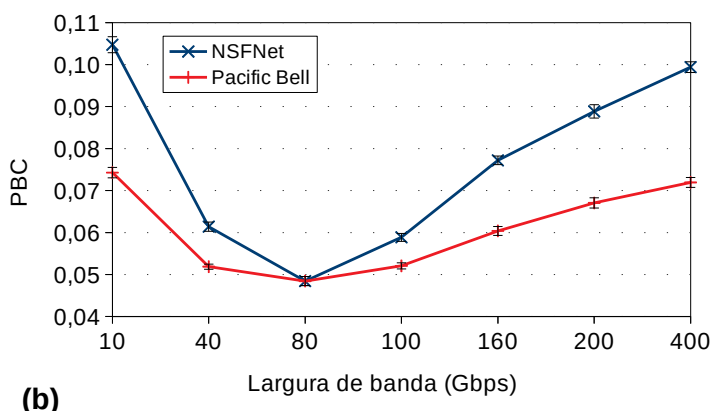

(b)

Figura 6. Estudos realizados com os algoritmos (a) DAFLF e (b) FirstLastFit.

Na Figura 6(b) são apresentadas as PBC alcançadas com o FLF nas topologias NSFNet e Pacific Bell em função do valor mínimo de largura de banda (parâmetro interno do FLF) utilizado para a seleção da partição de espectro (explicado na Seção 3). Notase pela Figura 6(b) que o algoritmo FLF apresenta o melhor desempenho em termos de PBC em ambas as topologias quando está usando a largura de banda de $80 \mathrm{Gbps}$ para o mecanismo de seleção de partição. A seleção da largura de banda de $80 \mathrm{Gbps}$ é justificada pela geração proporcional das granularidades das larguras de banda. No cenário considerado neste estudo são geradas mais requisições de circuitos com larguras de banda de 10 e 40 Gbps. A largura de banda de 80 Gbps também foi utilizada para o mecanismo de seleção (larger Band) do algoritmo SAIR.

A Figura 7 apresenta PBC, PBB e TMFE obtidas pelos algoritmos de alocação de espectro em função da carga na rede para a topologia NSFNet. O algoritmo SAIR apresentou melhor desempenho em termos de PBC e PBB em relação aos outros algoritmos para todos os valores de carga analisados, Figuras 7(a) e 7(b), respectivamente. Considerando a carga de 400 Erlangs, o algoritmo SAIR alcançou um ganho em termos de PBC relação aos algoritmos FF, BF, EF, RF, FLF, FLEF, TBSA e DAFLF de aproximadamente 
$89,17 \%, 86,58 \%, 79,74 \%, 72,97 \%, 76,57 \%, 79,66 \%, 74,38 \%$ e $80,19 \%$, respectivamente. Em termos de PBB, considerando a carga de 400 Erlangs, o algoritmo SAIR alcançou um ganho de aproximadamente 69,11\%, 67,19\%, 62,66\%, 56,45\%, 41,29\%, 62,63\%, 64,71\% e 52,36\% em relação aos algoritmos FF, BF, EF, RF, FLF, FLEF, TBSA e DAFLF, respectivamente.

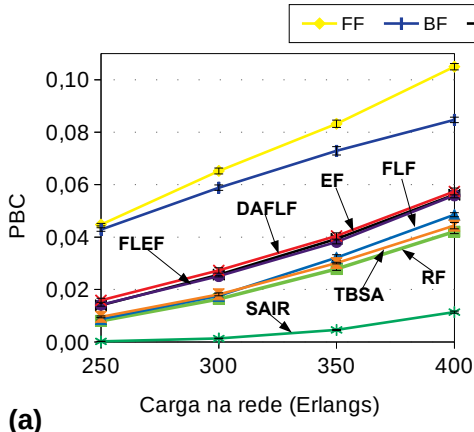

(a)

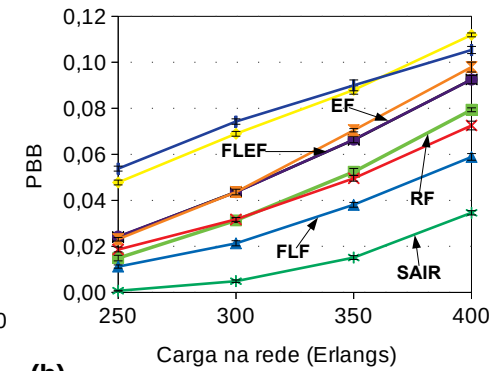

(b)

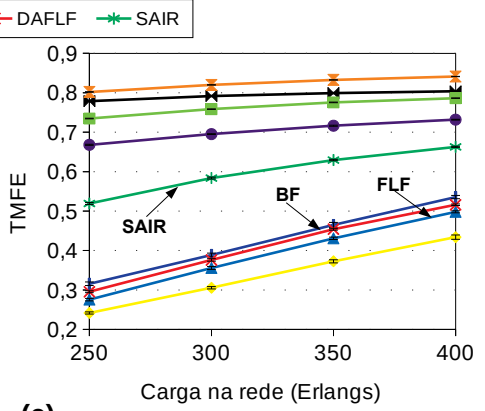

(c)

Figura 7. (a) Probabilidades de bloqueio de circuito, (b) Probabilidade de bloqueio de banda e (c) Taxa média de fragmentação externa obtidas pelos algoritmos de alocação de espectro para a topologia NSFNet.

Ainda analisando a Figura 7. A estratégia utilizada pelo RF diminuiu as interferências entre os circuitos, o que contribuiu para uma menor PBC em relação a maioria dos outros algoritmos, em compensação gerou uma alta TMFE (acima de 70\% para todas as cargas analisadas). O algoritmo TBSA apresentou um comportamento semelhante ao RF, menor PBC em relação a maioria dos outros algoritmos e alta TMFE. Entre os algoritmos que utilizam partições de espectro o FLEF apresentou os piores desempenhos em PBB e TMFE. As baixas TMFE (abaixo de 60\% para todas as cargas analisadas) alcançadas pelos algoritmos FF e BF devem-se ao fato deles possuírem as piores $\mathrm{PBC}$ e PBB. Já os algoritmos FLF e DAFLF possuem PBC e PBB menores que as PBC e PBB dos algoritmos FF e BF, e alcançaram baixas TMFE. O algoritmo SAIR apresentou as melhores PBC e PBB em relação aos outros algoritmos sem apresentar altas TMFE.

A Tabela 2 exibe as componentes da PBC obtidas com os algoritmos de alocação de espectro em função da carga na rede para a topologia NSFNet. As componentes da PBC consideradas neste trabalho são: fragmentação, AEL (ausência de espectro livre), QoTN (QoT inaceitável para o novo circuito) e QoTO (QoT inaceitável para um ou mais circuitos já ativos na rede).

Nota-se pela Tabela 2 que a componente que mais impactou nos desempenhos dos algoritmos FF, BF, EF, RF, FLF, FLEF, TBSA e DAFLF na topologia NSFNet foi a QoTO (destacada em negrito e em vermelho). Já a componente que mais impactou no desempenho do algoritmo SAIR na NSFNet foi a QoTN. A estratégia de buscar faixas de espectro que causem menos interferências nos outros circuitos evitou que o algoritmo SAIR sofresse bloqueios por QoTO. Isso representou uma redução considerável da probabilidade de bloqueio geral do algoritmo SAIR em relação aos outros algoritmos.

A Figura 8 apresenta PBC, PBB e TMFE obtidas pelos algoritmos de alocação de espectro em função da carga na rede para a topologia Pacific Bell. Nota-se pela Figura 8 que o desempenho do algoritmo SAIR na topologia Pcific Bell foi semelhante ao da NSFNet. O algoritmo SAIR obteve o melhor desempenho em termos de PBC e PBB em 
Tabela 2. Componentes da PBC obtidas pelos algoritmos de alocação de espectro para a topologia NSFNet na carga de $\mathbf{4 0 0}$ Erlangs.

\begin{tabular}{|c|cccc|}
\cline { 2 - 5 } \multicolumn{1}{c|}{} & Fragmentação & AEL & QoTN & QoTO \\
\hline FF & $0,034 \%$ & $0,003 \%$ & $0,625 \%$ & $\mathbf{9 9 , 3 3 8 \%}$ \\
\hline BF & $0,054 \%$ & $0,014 \%$ & $0,914 \%$ & $\mathbf{9 9 , 0 1 9 \%}$ \\
\hline EF & $0,086 \%$ & $0,002 \%$ & $11,424 \%$ & $\mathbf{8 8 , 4 8 8 \%}$ \\
\hline RF & $1,140 \%$ & $0,002 \%$ & $32,307 \%$ & $\mathbf{6 6 , 5 5 1 \%}$ \\
\hline FLF & $0,167 \%$ & $0,002 \%$ & $3,743 \%$ & $\mathbf{9 6 , 0 8 8 \%}$ \\
\hline FLEF & $0,097 \%$ & $0,009 \%$ & $11,815 \%$ & $\mathbf{8 8 , 0 7 9 \%}$ \\
\hline TBSA & $2,044 \%$ & $0,000 \%$ & $40,211 \%$ & $\mathbf{5 7 , 7 4 5 \%}$ \\
\hline DAFLF & $0,224 \%$ & $0,033 \%$ & $4,218 \%$ & $\mathbf{9 5 , 5 2 6 \%}$ \\
\hline SAIR & $12,240 \%$ & $0,234 \%$ & $\mathbf{8 7 , 5 2 6 \%}$ & $0,000 \%$ \\
\hline
\end{tabular}

relação aos outros algoritmos para todos os valores de carga analisados, Figuras 8(a) e 8(b). Considerando a carga de 400 Erlangs, o ganho do algoritmo SAIR em termos de PBC em relação aos algoritmos FF, BF, EF, RF, FLF, FLEF, TBSA e DAFLF foi de aproximadamente $55,73 \%, 48,92 \%, 42,53 \%, 35,73 \%, 28,92 \%, 40,77 \%, 41,10 \%$ e $29,82 \%$, respectivamente. Sob a carga de 400 Erlangs, o ganho do algoritmo SAIR em termos de PBB em relação aos algoritmos FF, BF, EF, RF, FLF, FLEF, TBSA e DAFLF foi de aproximadamente $29,99 \%, 30,77 \%, 39,60 \%, 40,01 \%, 3,63 \%, 38,80 \%, 51,46 \%$ e $15,33 \%$, respectivamente.

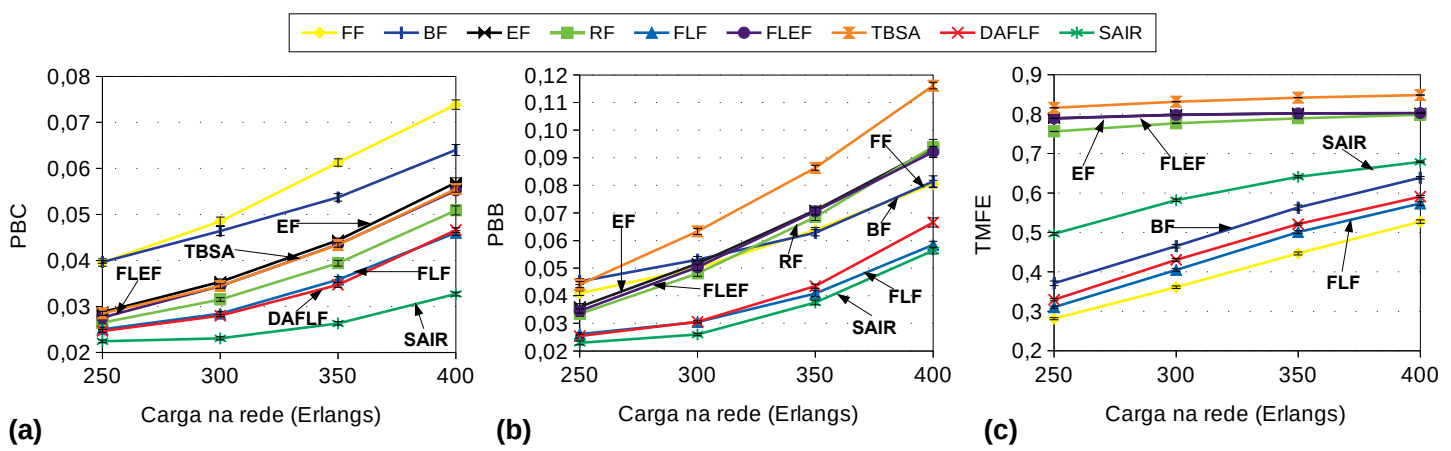

Figura 8. (a) Probabilidades de bloqueio de circuito, (b) Probabilidade de bloqueio de banda e (c) Taxa média de fragmentação externa obtidas pelos algoritmos de alocação de espectro para a topologia Pacific Bell.

Ainda analisando a Figura 8. Para o cenário da topologia Pacific Bell, os algoritmos RF e TBSA apresentam uma piora de desempenho em termos de PBC e PBB em relação aos outros algoritmos, quando comparados com os desempenhos apresentados na topologia NSFNet. Em termos de TMFE, os algoritmos de alocação de espectro mantiveram seus comportamentos apresentados na topologia NSFNet. $\mathrm{O}$ algoritmo SAIR manteve as melhores PBC e PBB sem apresentar altas TMFE (abaixo de 70\% para todas as cargas analisadas).

A Tabela 3 exibe as componentes da PBC obtidas com os algoritmos de alocação de espectro em função da carga na rede para a topologia Pacific Bell. Observa-se pela Tabela 3 que ocorreu um aumento na contribuição da componente QoTN na PBC de todos os algoritmos na topologia Pacific Bell. Isso ocorreu porque a solução de rotas encontrada 
pelo Dijkstra para a topologia Pacific Bell apresentou muitos enlaces gargalos. O que tornou mais escassas as faixas de slots livres que possibilitassem estabelecer circuitos com a QoT aceitável. Nota-se pela Tabela 3 que a ausência de bloqueios QoTO para o algoritmo SAIR, assim como na topologia NSFNet, contribui para o seu melhor desempenho em relação aos outros algoritmos na topologia Pacific Bell.

Tabela 3. Componentes da PBC obtidas pelos algoritmos de alocação de espectro para a topologia Pacific Bell na carga de 400 Erlangs.

\begin{tabular}{|c|cccc|}
\cline { 2 - 5 } \multicolumn{1}{c|}{} & Fragmentação & AEL & QoTN & QoTO \\
\hline FF & $0,130 \%$ & $0,016 \%$ & $33,194 \%$ & $\mathbf{6 6 , 6 6 0 \%}$ \\
\hline BF & $0,224 \%$ & $0,054 \%$ & $39,480 \%$ & $\mathbf{6 0 , 2 4 3 \%}$ \\
\hline EF & $0,247 \%$ & $0,000 \%$ & $\mathbf{5 6 , 2 4 0 \%}$ & $43,513 \%$ \\
\hline RF & $1,174 \%$ & $0,004 \%$ & $\mathbf{7 5 , 2 0 3 \%}$ & $23,619 \%$ \\
\hline FLF & $0,407 \%$ & $0,002 \%$ & $\mathbf{5 6 , 4 7 4 \%}$ & $43,116 \%$ \\
\hline FLEF & $0,258 \%$ & $0,011 \%$ & $\mathbf{5 8 , 0 4 1 \%}$ & $41,691 \%$ \\
\hline TBSA & $1,518 \%$ & $0,000 \%$ & $\mathbf{8 0 , 3 8 2 \%}$ & $18,100 \%$ \\
\hline DAFLF & $0,556 \%$ & $0,046 \%$ & $\mathbf{5 9 , 3 1 2 \%}$ & $40,086 \%$ \\
\hline SAIR & $1,809 \%$ & $0,072 \%$ & $\mathbf{9 8 , 1 1 9 \%}$ & $0,000 \%$ \\
\hline
\end{tabular}

Considerando o cenário apresentado na topologia NSFNet (os bloqueios por QoTO impactam mais no desempenho dos algoritmos). Dois algoritmos que não utilizam partições de espectro (RF e TBSA) conseguiram alcançar bons desempenhos de PBC e PBB (principalmente de PBC) em detrimento a TMFE. Já no cenário apresentado na topologia Pacific Bell (os bloqueios por QoTN impactam mais no desempenho dos algoritmos), os algoritmos que utilizam partições de espectro apresentaram os melhores desempenhos em PBC, PBB e TMFE. Dentre eles apenas o algoritmo FLEF não apresentou um bom desempenho nos cenários avaliados.

\section{Conclusão}

Este artigo propôs o algoritmo SAIR que realiza a alocação de espectro considerando imperfeições de camada física em redes ópticas elásticas. O objetivo do algoritmo SAIR é selecionar faixas de espectro livre que causem menos interferências nos outros circuitos já ativos na rede. Foi realizado um estudo de avaliação de desempenho comparado o algoritmo SAIR com os algoritmos FF, BF, EF, RF, FLF, FLEF, TBSA e DAFLF. O algoritmo DAFLF também é um algoritmo de alocação de espectro ciente dos efeitos de camada física. Os algoritmos foram comparados considerando as métricas de PBC, PBB e TMFE nas topologias NSFNet e Pacific Bell.

Os resultados do estudo de avaliação de desempenho mostraram que o algoritmo SAIR apresentou o melhor desempenho em termos de PBC e PBB em comparação aos outros algoritmos nas topologias NSFNet e Pacific Bell. Os resultados ainda mostram que o melhor desempenho do algoritmo SAIR em termos de PBC e PBB não foi em função de altas TMFE (acima de 70\%). Considerando a carga de 400 Erlangs na topologia NSFNet, o algoritmo SAIR apresentou ganhos mínimos de 72,97\% e 41,29\% em termos de PBC e PBB, respectivamente. E na topologia Pacific Bell, considerando a carga de 400 Erlangs, os ganhos mínimos alcançados foram $28,92 \%$ e 3,63\% em termos de PBC e $\mathrm{PBB}$, respectivamente. 


\section{Referências}

Beyranvand, H. and Salehi, J. (2013). A quality-of-transmission aware dynamic routing and spectrum assignment scheme for future elastic optical networks. Journal of Lightwave Technology, 31(18):3043-3054.

Chatterjee, B. C., Fadini, W., and Oki, E. (2016). A spectrum allocation scheme based on first-last-exact fit policy for elastic optical networks. Journal of Network and Computer Applications, 68(Supplement C):164 - 172.

Chatterjee, B. C. and Oki, E. (2016). Dispersion-adaptive first-last fit spectrum allocation scheme for elastic optical networks. IEEE Communications Letters, 20(4):696-699.

Chatterjee, B. C., Sarma, N., and Oki, E. (2015). Routing and spectrum allocation in elastic optical networks: A tutorial. IEEE Communications Surveys Tutorials, 17(3):17761800 .

Chaves, D., Carvalho, R., Pereira, H., Bastos-Filho, C., and Martins-Filho, J. (2012). Novel strategies for sparse regenerator placement in translucent optical networks. Photonic Network Communications, 24(3):237-251.

Christodoulopoulos, K., Tomkos, I., and Varvarigos, E. (2011). Elastic bandwidth allocation in flexible OFDM-based optical networks. Journal of Lightwave Technology, 29(9):1354-1366.

Fontinele, A., Santos, I., Neto, J. N., Campelo, D. R., and Soares, A. (2017). An efficient IA-RMLSA algorithm for transparent elastic optical networks. Computer Networks, 118(Supplement C):1 - 14 .

Leiva, A., Pavez, N., Beghelli, A., and Olivares, R. (2014). A joint RSA algorithm for dynamic flexible optical networking. In 2014 IEEE Latin-America Conference on Communications (LATINCOM), pages 1-6.

Liu, H. L., Lv, L., Chen, Y., and Wei, C. (2017). Fragmentation-avoiding spectrum assignment strategy based on spectrum partition for elastic optical networks. IEEE Photonics Journal, 9(5):1-13.

Rosa, A., Cavdar, C., Carvalho, S., Costa, J., and Wosinska, L. (2012). Spectrum allocation policy modeling for elastic optical networks. In High Capacity Optical Networks and Emerging/Enabling Technologies, pages 242-246.

Saradhi, C. V. and Subramaniam, S. (2009). Physical layer impairment aware routing (PLIAR) in WDM optical networks: Issues and challenges. Commun. Surveys Tuts., 11(4):109-130.

Talebi, S., Alam, F., Katib, I., Khamis, M., Salama, R., and Rouskas, G. N. (2014). Spectrum management techniques for elastic optical networks: A survey. Optical Switching and Networking, 13:34 - 48 .

Wu, J., Ning, Z., and Guo, L. (2017). Energy-efficient survivable grooming in softwaredefined elastic optical networks. IEEE Access, 5:6454-6463.

Zhu, P., Li, J., Wu, D., Chen, Y., Tian, Y., Wu, Z., Ge, D., Chen, X., Chen, Z., and He, Y. (2017). Software-defined elastic optical network node supporting spectrum defragmentation. IEEE/OSA Journal of Optical Communications and Networking, 9(1):A63A70. 